\title{
Management of diabetic patients with aortic intramural hematoma: There may be more involved besides MMP9
}

\author{
Arminder Jasser ${ }^{1}$ and Gus Vlahakes ${ }^{1}$ \\ ${ }^{1}$ Massachusetts General Hospital
}

June 11, 2020

\begin{abstract}
This Editorial Comment explores important issues raised by this manuscript.
\end{abstract}

Is intramural hematoma, in fact, an aortic dissection where the intimal tear is not visualized by the current imaging technology? There is certainly a divide between the east and the west regarding the appropriate management strategy for intramural hematoma involving the ascending aorta (IMHA). In the United states, an IMHA is mostly treated like a Type A dissection, with urgent surgery. However, several reports from Asian centers, such as the current series, propose an initial medical approach constituting impulse control and serial imaging to select patients for surgical treatment. These reports have enhanced our understanding of high risk features surrounding IMH (pericardial effusion, aortic diameter $>5 \mathrm{~cm}$, or IMH width $>1 \mathrm{~cm}$ ) that will likely fail medical management and require surgery.

In this manuscript, the authors have compared outcomes of medical management of IMHA in patients with newly diagnosed DM to those without DM and report a higher rate of death associated with increased MMP9 levels in patients without DM. The authors contribute the early mortality in the DM group to a sharp increase in the MMP9 levels due to aggressive glycemic control in this group, often with use of insulin. It is however, interesting to note that the only time period with significant difference in mortality between the two groups, with higher mortality in patients without DM is during the 3-6 month period (Figure 4D), when the MMP 9 levels patients without DM are rapidly declining, which is not congruent with the hypothesis of the study. Further, the only mortality in the 25-36 months is in the DM group; during this period, the MMP9 levels in this group are significantly lower as compared to the no DM group. Also, the authors report that 8 of 10 late deaths occurred after ascending aortic complications (retrograde Type A dissection or ascending aortic pseudoaneurysms) after TEVAR placement. This is very high, likely related to placing a stent graft with the proximal landing zone in diseased aorta, something that we try hard to avoid while treating patients with acute Type B dissections, taking measures (arch re-routing etc.) to ensure a proximal stent landing zone in undissected aorta. In patients with IMHA, that is not possible due to the hematoma extending into the ascending aorta. The outcomes in the group of patients subjected to this approach in the current study raises the question if these complications are treatment related, as much as disease related.

While there remain some questions regarding the findings of the current report, if one were to believe the conclusions of the study, an even more interesting question comes up - how aggressively should DM be controlled in patients with IMHA, or more broadly, with all acute aortic syndromes? And for how long? The systemic manifestations of DM are well known and the potential protective effect of DM for aortic disease presents an important clinical conundrum for the clinicians providing care for these patients; it runs counterintuitive to all we have learned about patient care. The presence or absence of DM is an unmodifiable 
risk factor, particularly when newly diagnosed in a patient presenting with an IMHA. The only modifiable factor is how we treat it. There is an interesting mechanism question that has not been answered by any study, but which is suggested by the authors' findings: Could some IMH arise from intramural bleeding from vasa vasora, rather than from a localized dissection not detected by imaging. If DM can affect vasa vasora so that the microvascular anatomy of the aorta is diminished, could this result in selection of patients with smaller IMH?

Perhaps future studies examining aortic outcomes in patients with DM and detailed pathologic examinations, comparing patients with strict or relaxed glycemic control will help answer some of these questions.

1. Evangelista A, Mukherjee D, Mehta RH, et al. Acute intramural hematoma of the aorta: a mystery in evolution. Circulation 2005;111:1063-70. [Crossref] [PubMed]

2. Estrera A, Miller C 3rd, Lee TY, et al. Acute type A intramural hematoma: analysis of current management strategy. Circulation 2009;120:S287-91. [Crossref] [PubMed]

3. Harris KM, Braverman AC, Eagle KA, et al. Acute aortic intramural hematoma: an analysis from the International Registry of Acute Aortic Dissection. Circulation 2012;126:S91-6. [Crossref] [PubMed]

4. Kaji S, Nishigami K, Akasaka T, et al. Prediction of progression or regression of type A aortic intramural hematoma by computed tomography. Circulation 1999;100:II281-6. [Crossref] [PubMed]

5. Kitai T, Kaji S, Yamamuro A, et al. Clinical outcomes of medical therapy and timely operation in initially diagnosed type a aortic intramural hematoma: a 20-year experience. Circulation 2009;120:S292-8. [Crossref $][$ PubMed $]$

6. Song JK, Yim JH, Ahn JM, et al. Outcomes of patients with acute type a aortic intramural hematoma. Circulation 2009;120:2046-52. [Crossref] [PubMed]

Note that mortality rates of medically-treated patients in the European and American series are higher (1-3), compared with the Asian series (4-6). 